\title{
Protective effects of gastrodin pretreatment on mouse hepatic ischemia-reperfusion occurring through antioxidant and anti-apoptotic mechanisms
}

\author{
BO SUN $^{1 *}$, JIE JIANG $^{1 *}$, XINYAN ZHU ${ }^{2 *}$, DAN YANG ${ }^{1}$, ZHENYU CUI $^{1}$, YU ZHANG $^{1}$, \\ MINBO ZHANG ${ }^{1}$, YITING QIAN ${ }^{1}$, RUILIN LIU $^{3}$ and WENZHUO YANG ${ }^{1}$ \\ ${ }^{1}$ Department of Gastroenterology and Hepatology, Institute of Digestive Disease, Tongji Hospital, \\ Tongji University School of Medicine, Shanghai 200065; 2 Department of Gastroenterology, Shanghai East Hospital, \\ Tongji University School of Medicine, Shanghai 200120; ${ }^{3}$ Department of Pulmonary and Critical Care Medicine, \\ Tongji Hospital, Tongji University School of Medicine, Shanghai 200065, P.R. China
}

Received March 26, 2020; Accepted February 8, 2021

DOI: $10.3892 /$ etm.2021.9902

\begin{abstract}
Hepatic ischemia-reperfusion injury (HIRI) often occurs following surgical procedures such as liver resection and transplantation. However, despite its clinical prominence, to the best of our knowledge, there remain no effective strategies to treat HIRI. Therefore, the aim of present study was to identify therapeutic agents that can exert beneficial effects against HIRI. The present study found that following hepatic IR modeling in mice, gastrodin (Gas) pretreatment improved the IR outcomes in terms of the serum biochemical indexes (alanine transaminase and aspartate transaminase), tissue biochemical indexes (superoxide dismutase, malondialdehyde and reduced glutathione content) and tissue pathology (H\&E staining). In addition, compared with those in the IR + vehicle group, the IR + Gas group showed upregulated expression levels of nuclear erythroid 2-related factor 2, heme oxygenase 1 and $\mathrm{Bcl}-2$ as detected by western blotting and reverse transcription-quantitative PCR. The mRNA and protein expression levels of Bax and caspase-3 were downregulated in the IR + Gas group compared with the IR + vehicle group. Concurrently, no significant differences were observed in the parameters between the Sham + vehicle and the Sham + Gas groups, indicating that Gas pretreatment
\end{abstract}

Correspondence to: Dr Ruilin Liu, Department of Pulmonary and Critical Care Medicine, Tongji Hospital, Tongji University School of Medicine, 389 Xincun Road, Shanghai 200065, P.R. China

E-mail: 18721881628@163.com

Dr Wenzhuo Yang, Department of Gastroenterology and Hepatology, Institute of Digestive Disease, Tongji Hospital, Tongji University School of Medicine, 389 Xincun Road, Shanghai 200065, P.R. China E-mail: yangwenzhuo2002@163.com

${ }^{*}$ Contributed equally

Key words: hepatic ischemia-reperfusion, gastrodin, oxidative stress, apoptosis, nuclear factor erythroid 2-related factor 2 may not cause liver damage. In conclusion, the findings of the present study revealed that Gas pretreatment exerted a protective effect in HIRI through both antioxidant and anti-apoptotic mechanisms.

\section{Introduction}

Hepatic ischemia-reperfusion injury (HIRI) generally occurs during hemorrhagic shock, hepatectomy and liver transplantation (1). In the United States, liver transplantation accounted for $23 \%$ transplant procedures in 2015, where one of the most formidable barriers to successful liver transplantation is ischemia-reperfusion injury (2). However, despite the prominent clinical presence of HIRI, there are currently no effective drugs available to prevent IR injury.

During hepatic IR, liver injury caused by hypoxia is further exacerbated by the restoration of blood flow (3). A large number of free radicals and reactive oxygen species produced as a result of IR, including superoxide, hydrogen peroxide and hydroxyl radicals, have been shown to exert serious deleterious effects on hepatocytes $(4,5)$. During the oxidative stress reaction, the nuclear erythroid 2-related factor 2 (Nrf2)/heme oxygenase (HO)-1 pathway is one of the most important antioxidant systems (6). It has also been reported that apoptosis induced by mitochondrial permeability transition (MPT) leads to caspase (CASP) activation and that MPT also serves an important role in the pathogenesis of IR injury (7). Therefore, it was hypothesized that suppressing apoptosis and oxidative stress following acute injury may be key to treating hepatic IR.

Gastrodin is an organic compound that can be extracted from the dried root blocks of the orchid plant Gastrodia elata Blume, which has a long history of clinical application in Chinese medicine $(8,9)$. Gastrodin (Gas; PubChem CID, 115027; Fig. S1) is the major active ingredient in Rhizome Gastrodiae. According to previous studies, Gas has been found to protect liver cells, neurons and cardiomyocytes by inhibiting oxidative stress and parenchymal apoptosis $(4,10)$; however, to the best of our knowledge, no studies have investigated its role in hepatic IR. As such, regarding the potential 
application of Gas, the present study aimed to investigate the role and mechanism of action of Gas pretreatment at various times during hepatic IR in mice by constructing a mouse hepatic IR model.

\section{Materials and methods}

Animal studies. A total of 48 6-week-old male (20-23 g) C57/BL6 mice were purchased from Shanghai Slark Laboratory Animal Co., Ltd. The animals were housed in a pathogen-free animal room at the Hubei Animal Experiment Center of Tongji University at $25 \pm 3^{\circ} \mathrm{C}$ and $55 \pm 5 \%$ humidity, with a 12-h light/dark cycle, a standard experimental diet and free access to drinking water. Forty-eight C57/BL6 mice were randomly divided into four groups (12 mice/group): i) Gas (cat. no. 62499-27-8; MedChemExpress) pretreatment + hepatic IR model (IR + Gas group), whereby $300 \mathrm{mg} / \mathrm{kg} / \mathrm{day}$ Gas, the dose reported in the literature (4), was continuously administered into the stomach for 8 days prior to the establishment of the hepatic IR model; ii) hepatic IR model (IR + vehicle group), in which an equal volume of normal saline was administered to the stomach for 8 consecutive days prior to the establishment of the hepatic IR model; iii) Gas pretreatment + sham group (Sham + Gas group), whereby Gas was continuously administered to the stomach for 8 days, then the abdominal cavity was opened and the portal vein, hepatic vein and hepatic artery were simply separated to simulate a sham operation; and iv) sham operation group (Sham + vehicle group), whereby an equal volume of normal saline was administered to the stomach for 8 days prior to the sham operation. Following 2 and $24 \mathrm{~h}$ of modeling, six mice were randomly selected from each group. The mice were euthanized by overdoes of inhalant anesthetics, followed by exsanguination under anesthesia to ensure death. When the mice showed no respiration, no heartbeat and no response to any external stimuli, they were considered dead. Samples required for testing were collected.

Establishment of hepatic IR model. Prior to the experimental procedure, the mice were fasted for $12 \mathrm{~h}$ and were kept water-free for $4 \mathrm{~h}$. The model of hepatic IR in male mice was established using the Pringle's method (11). The mice were anesthetized by inhalation of isoflurane (oxygen flow rate, $1.0 \mathrm{l} / \mathrm{min}$; induction dose, $3 \%$; maintenance dose, $2 \%$ ). Then the mice were placed on the operating table and the abdominal cavity and liver was exposed along the white line of the abdomen. Microvascular forceps were used to clamp the vessels in the left and middle lobe of the liver, causing 70\% liver ischemia. The color of the left lobe liver and middle lobe liver tissue immediately changed from reddish brown to light red. Following $60 \mathrm{~min}$ of ischemia, the microvascular forceps were removed to restore the liver blood supply and at the same time, restore the anatomical position of the abdominal organs. The abdominal wall was sutured with double layers and the mouse liver IR model was successfully constructed.

$H \& E$ staining. Liver segments from the left lobe of the liver from each group of mice were cut, fixed with $4 \%$ paraformaldehyde for $24 \mathrm{~h}$ at room temperature, routinely dehydrated at room temperature using an increasing ethanol gradient followed by xylene. The sections were then embedded in paraffin and cut into 5-mm-thick tissue sections. The sections were subsequently stained with $\mathrm{H} \& \mathrm{E}$ (hematoxylin was stained for $5 \mathrm{~min}, 1 \% \mathrm{HCl}$ ethanol for 1-3 sec and eosin staining for $3 \mathrm{~min}$, all at room temperature) and visualized using a Nikon light microscope at $\mathrm{x} 400$ magnification (Nikon Corporation). The severity of liver pathological damage was evaluated using the Suzuki standard method (Table SI) (12). Three experienced pathologists scored each sample in a blinded manner.

Tissue and serum indicators. Serum was obtained at 2 and $24 \mathrm{~h}$ following the operation. Alanine transaminase (ALT) and aspartate transaminase (AST) levels in the serum were detected using ALT and AST kits (cat. nos. C009-2-1 and C010-2-1, respectively; Nanjing Jiancheng Bioengineering Institute), according to the manufacturer's protocol. The content of superoxide dismutase (SOD), malondialdehyde (MDA) and glutathione (GSH) in the liver tissues was analyzed using SOD, MDA and GSH kits, respectively (cat. nos. A001-3-2, A003-1-2 and A006-2-1, respectively; Nanjing Jiancheng Bioengineering Institute).

Reverse transcription-quantitative PCR (RT-qPCR). Total RNA was extracted from the liver tissues of each group of mice using TRIzol ${ }^{\circledR}$ reagent (Invitrogen; Thermo Fisher Scientific, Inc.). Total RNA was reverse transcribed into cDNA using PrimeScript ${ }^{\mathrm{TM}}$ RT Master Mix (Perfect Real Time; cat. no. RR036B; Takara Bio, Inc.). The temperature protocol were as follows: $37^{\circ} \mathrm{C}$ for $15 \mathrm{~min}, 85^{\circ} \mathrm{C}$ for $5 \mathrm{sec}$. RT-qPCR was subsequently performed using TB Green ${ }^{\circledR}$ Premix Ex Taq ${ }^{\mathrm{TM}}$ (cat. no. RR042B; Takara Bio, Inc.). The full thermocycling conditions for qPCR are as follows: Initial denaturation at $95^{\circ} \mathrm{C}$ for $30 \mathrm{sec}$, followed by 40 cycles of $95^{\circ} \mathrm{C}$ for $5 \mathrm{sec}$ and $60^{\circ} \mathrm{C}$ for $30 \mathrm{sec}$. The primer sequences are presented in Tables SII and SIII. Expression levels were quantified using the $2^{-\Delta \Delta \mathrm{Ca}}$ method (13) and normalized to $\beta$-actin.

Western blotting. Western blotting was performed RIPA lysis buffer (Beyotime Institute of Biotechnology) from liver specimens. Equal amounts of protein $(30 \mu \mathrm{g}$; quantified using the bicinchoninic acid protein assay) were separated using $10 \%$ SDS-PAGE and then wet-transferred onto a PVDF membrane (cat. no. IPVH00010; EMD Millipore). The membranes were blocked with $5 \%$ non-fat milk for $1 \mathrm{~h}$ at room temperature and subsequently incubated with the following primary antibodies at $4^{\circ} \mathrm{C}$ overnight: Anti-Nrf2 (cat. no. 16396-1-AP; 1:1,000; ProteinTech Group, Inc.), anti-HO-1 (cat. no. 10701-1-AP; 1:1,000; ProteinTech Group, Inc.), anti-Bax (cat. no. ab32503; 1:1,000; Abcam), anti-Bcl-2 (cat. no. ab32124; 1:1,000; Abcam), anti-CASP-3 (cat. no. ab32351; 1:1,000; Abcam) and anti- $\beta$-actin (cat. no. MA1-140; $1: 5,000$; Thermo Fisher Scientific, Inc.). Following the primary antibody incubation, the membranes were incubated with the horseradish peroxidase-conjugated goat anti-rabbit IgG secondary antibody (cat. no. A0208; 1:10,000; Beyotime Institute of Biotechnology) and goat anti-mouse IgG (cat. no. A0216; 1:10,000; Beyotime Institute of Biotechnology) at room temperature for $1 \mathrm{~h}$. The protein bands were visualized by BeyoECL Plus (Beyotime Institute of Biotechnology). QuantityOne v4.6.6 software (Bio-Rad Laboratories, Inc.) was used for analysis and the ratio of the gray value of the target protein band to the gray 
value of the internal reference $\beta$-actin protein band was used to represent the relative expression levels of the target protein.

Statistical analysis. GraphPad Prism software (GraphPad Software, Inc.) and SPSS 19.0 software (IBM Corp.) were used for statistical analysis. One-way ANOVA, followed by Bonferroni's correction or Tamhane's T2 post-hoc test, was used to determine the statistical differences between groups. A Kruskal Wallis test, followed by post-hoc Dunn's test, was used to evaluate non-parametric data. All data are presented as the mean \pm SEM, or median (25-75th percentile). $\mathrm{P}<0.05$ was considered to indicate a statistically significant difference.

\section{Results}

Gas pretreatment alleviates the degree of liver IR injury in mice. H\&E staining and a Suzuki's score were used to evaluate the liver histopathological changes of mice in each group. H\&E pathological sections revealed that the Sham + Gas and Sham + vehicle groups had complete hepatic lobules without the presence of abnormal inflammatory cell infiltration, fragments or massive necrosis (Fig. 1A). No significant differences were observed in the Suzuki's score (Table I) and in serum ALT and AST levels (Fig. 1B).

The pathological changes in the IR groups appeared to be different. Compared with the IR + vehicle group, the liver pathological changes, such as liver congestion, vacuole-like changes and necrosis, were markedly alleviated in the IR + Gas group at 2 and $24 \mathrm{~h}$ (Fig. 1A). Following Gas pretreatment, the pathological results of the IR + Gas group revealed that the structure of the liver lobules was intact, the red blood cell depositions in the central vein and hepatic sinuses were reduced, and the proportion of cytoplasmic vacuoles and necrotic cells were reduced. The IR + Gas group displayed a lower Suzuki's score and decreased serum levels of ALT and AST at $2 \mathrm{~h}$ post-modeling, with the trends being the same at $24 \mathrm{~h}$ (Fig. 1; Table I).

Pathological and serological results confirmed that Gas pretreatment itself did not cause damage to the liver tissue and function. Gas pretreatment appeared to have reduced the occurrence of congestion, vacuole-like changes and necrosis following hepatic IR. Therefore, subsequent experiments focused on the effect of Gas pretreatment on mice hepatic IR.

Gas pretreatment enhances the ability ofmice to resist oxidative stress. Oxygen free radicals can attack polyunsaturated fatty acids in biofilms, decompose lipid hydroperoxides and damage liver tissue (14-16). Following hepatic IR modeling, due to the effect of Gas, the liver SOD activity in the IR + Gas group was significantly increased at both the 2 and $24 \mathrm{~h}$ time-points, while the MDA content was significantly decreased compared with that in the IR + vehicle group (Fig. 2). In addition, compared with those in the IR + vehicle group, liver GSH content in the IR + Gas group showed an upward trend at the time-point of $2 \mathrm{~h}$ without significant difference, but increased significantly at the time-point of $24 \mathrm{~h}$.

To investigate whether Gas pretreatment enhanced the antioxidant capacity of normal mice, the Sham + vehicle group was compared with the Sham + Gas group. The results confirmed that the liver GSH content in the Sham + Gas group was significantly higher compared with that in the
Table I. Suzuki's score of each group at different time-points.

Suzuki's score

\begin{tabular}{lcc}
\cline { 2 - 3 } Group & $2 \mathrm{~h}$ & $24 \mathrm{~h}$ \\
\hline Sham + vehicle group & $0.00(0.00-1.00)$ & $0.50(0.00-1.00)$ \\
Sham + Gas group & $0.00(0.00-1.00)$ & $1.00(0.00-1.00)$ \\
IR + vehicle group & $6.50(5.50-8.00)^{\mathrm{a}}$ & $7.00(6.75-8.00)^{\mathrm{a}}$ \\
IR + Gas group & $4.00(4.00-6.00)^{\mathrm{b}}$ & $5.50(5.00-7.00)^{\mathrm{b}}$
\end{tabular}

Data are presented as the median (25-75th percentile; $\mathrm{n}=6$ mice/group at each time-point). ${ }^{\mathrm{a}} \mathrm{P}<0.001$ vs. the Sham + vehicle group; ${ }^{\mathrm{b}} \mathrm{P}<0.01$ vs. the IR + vehicle group. Gas, gastrodin; IR, ischemic-reperfusion group.

Sham + vehicle group (Fig. 2). Although there were no differences observed in SOD activity and MDA content between the Sham groups; however, they were significantly changed following HIRI. The expression levels of the SOD gene were also analyzed; the results revealed that compared with the IR + vehicle group, SOD expression levels were significantly increased following Gas pretreatment (Figs. 3B and 4B).

Gas pretreatment upregulates $\mathrm{Nrf} 2$ and $\mathrm{HO}-1$ expression levels in the liver. Subsequently, the present study investigated whether Gas pretreatment affected the expression levels of liver Nrf2 and HO-1.

At $2 \mathrm{~h}$ following hepatic IR modeling, compared with the IR + vehicle group, the HO-1 expression levels in the liver of the IR + Gas group were significantly upregulated; however, there were no significant differences observed between Nrf 2 mRNA and protein expression levels (Fig. 3A and B). However, at $24 \mathrm{~h}$ after hepatic IR modeling, the liver Nrf2 and HO-1 expression levels in the IR + Gas group demonstrated similar changes, with significantly upregulated expression levels (Fig. 4A and B).

The expression levels of Nrf2 appeared to fluctuate over time, which was not only observed between the IR groups, but also between the sham groups (Figs. 3A and 4A).

Gas pretreatment improves the antiapoptotic ability of the liver through regulating the expression levels of Bax, Bcl-2 and $C A S P-3$. The present study also investigated the expression levels of Bax, Bcl-2 and CASP-3 in the liver of each group of mice (Figs. 5 and 6). The results revealed that Gas significantly downregulated the expression levels of Bax and cleaved CASP-3, while upregulating Bcl-2 expression levels at 2 and $24 \mathrm{~h}$ post hepatic IR modeling (Figs. 5B and 6B). This trend was further confirmed at the protein expression level, as detected by western blotting (Figs. 5A and 6A). Meanwhile, no significant differences were observed between the Sham + vehicle and the Sham + Gas groups (Figs. 5 and 6). These results suggested that Gas pretreatment may not cause damage to normal mice liver tissue.

\section{Discussion}

Previous studies have reported that Gas improves the ability of cells to resist hypoxia and apoptosis, and exerts protective 
A
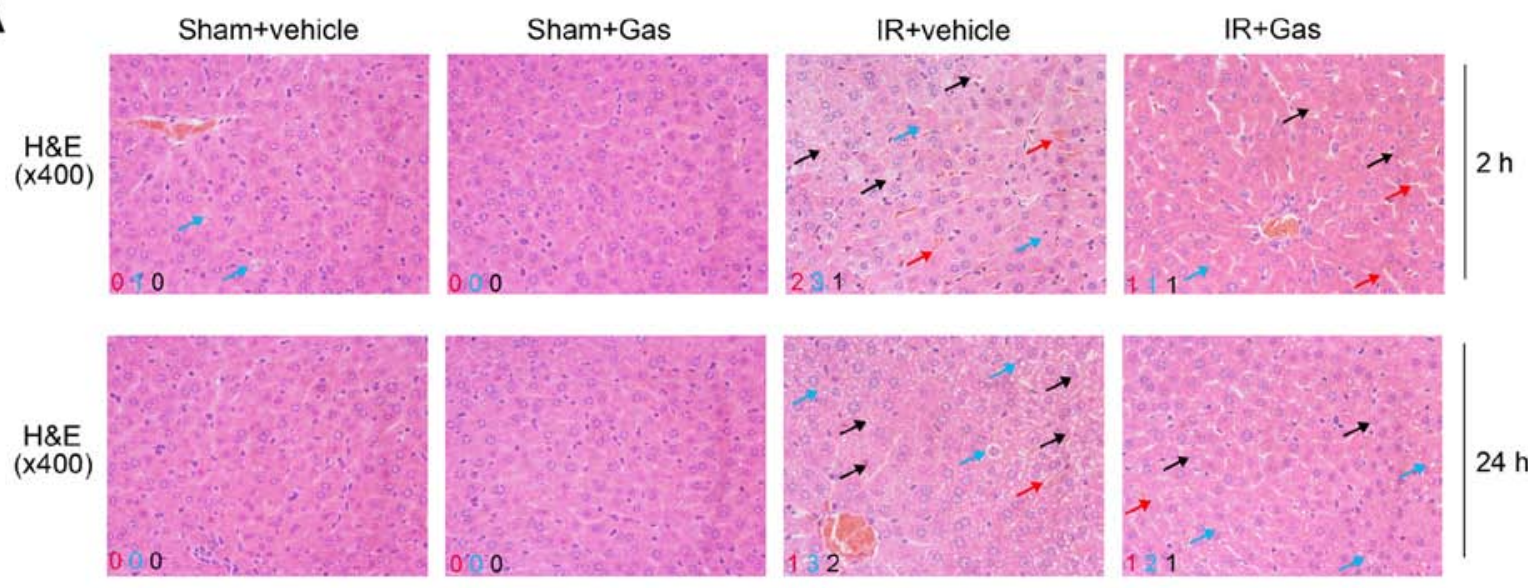

B

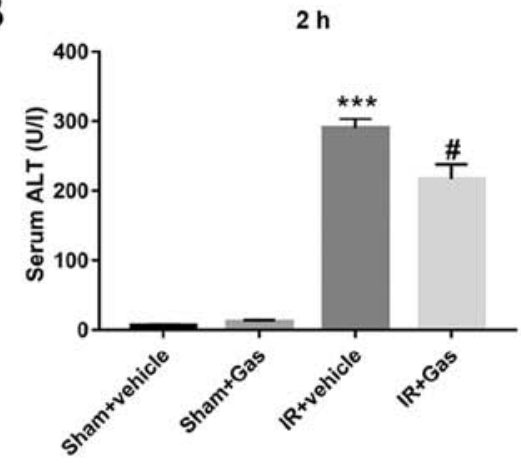

$24 \mathrm{~h}$

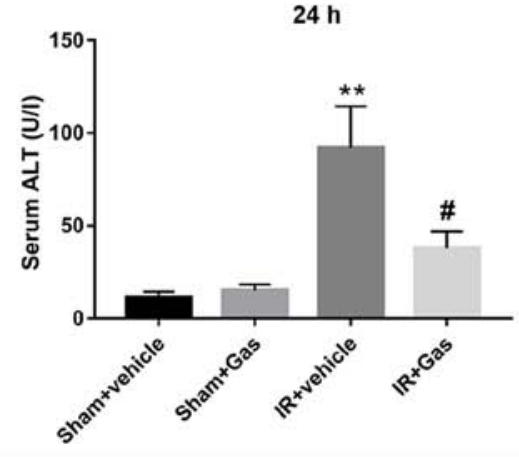

$2 \mathrm{~h}$

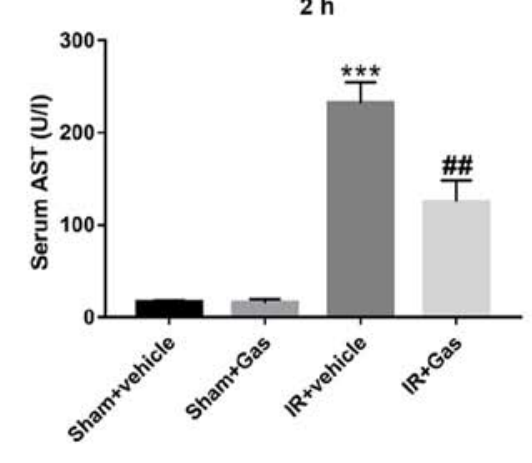

$24 \mathrm{~h}$

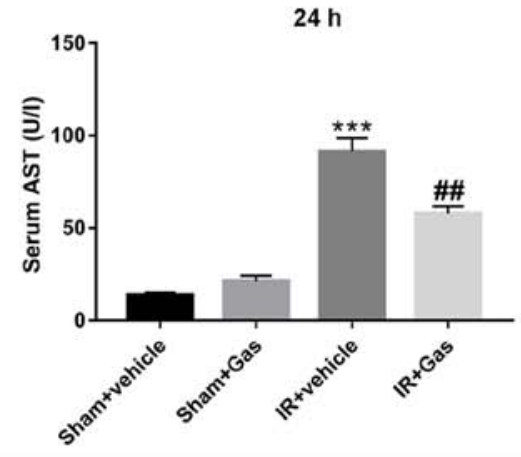

Figure 1. GAS alleviates the severity of liver injury induced by IR. (A) Representative images of H\&E staining of the liver sections following hepatic IR. Red arrows indicate congested areas, blue arrows indicate vacuolation areas and black arrows indicate necrotic areas. The numbers in the bottom left corner of each image indicate the Suzuki's scores. (B) Serum ALT and AST levels in mice at 2 and $24 \mathrm{~h}$ post-modeling ( $\mathrm{n}=6 \mathrm{mice}$ ). ${ }^{* *} \mathrm{P}<0.01$, ${ }^{* * *} \mathrm{P}<0.001 \mathrm{vs}$. the Sham + vehicle group; ${ }^{\#} \mathrm{P}<0.05,{ }^{\#} \mathrm{P}<0.01$ vs. the IR + vehicle group. GAS, gastrodin; ALT, alanine aminotransferase; AST, aspartate aminotransferase; $\mathrm{IR}$, ischemia-reperfusion.

effects on the mouse heart and following cerebral IR (4,17-19). As such, the present study aimed to determine the role of Gas in mice hepatic IR.

To determine the concentration and dosage of Gas pretreatment, previous literature reports were used (4). A concentration of $300 \mathrm{mg} / \mathrm{kg} /$ day was administered by gavage 8 days before modeling and the changes in the related indicators were analyzed at 2 and $24 \mathrm{~h}$. The results of the present study revealed IR caused significant pathological changes within the two time-points by the end of the model, indicating that the hepatic IR model was established successfully and that the liver tissue was severely damaged. Thus, the present study aimed to investigate the role of Gas preconditioning in HIRI.

Similar to previous studies, the levels of three biochemical indicators, SOD, GSH and MDA, were determined to evaluate the ability of antioxidative stress following Gas pretreatment (20). The experimental results confirmed that Gas pretreatment significantly enhanced the antioxidative stress capacity following hepatic IR in mice. Therefore, these findings suggested that Gas pretreatment may help the mouse liver to accelerate the elimination of superoxide anion free radicals, stabilize enzymes containing thiol groups and prevent hemoglobin and other cofactors from oxidative damage 
A

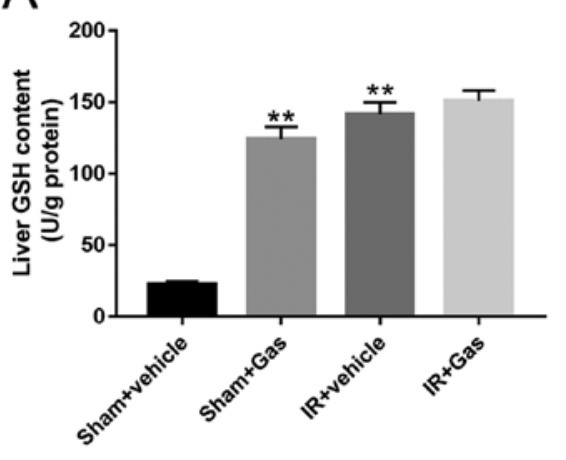

$2 \mathrm{~h}$

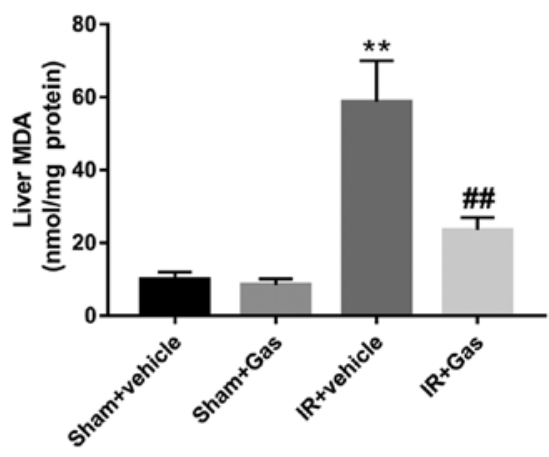

$2 \mathrm{~h}$

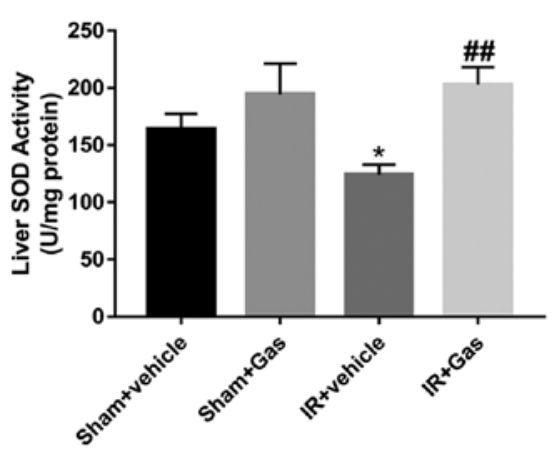

B

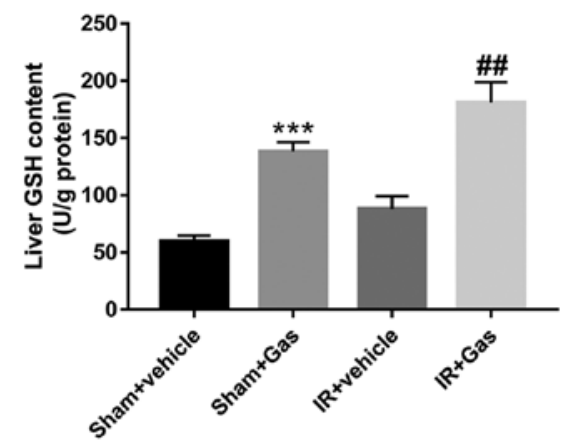

$24 \mathrm{~h}$

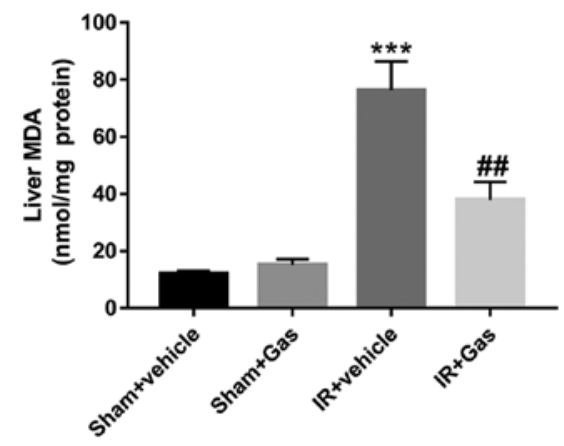

24h

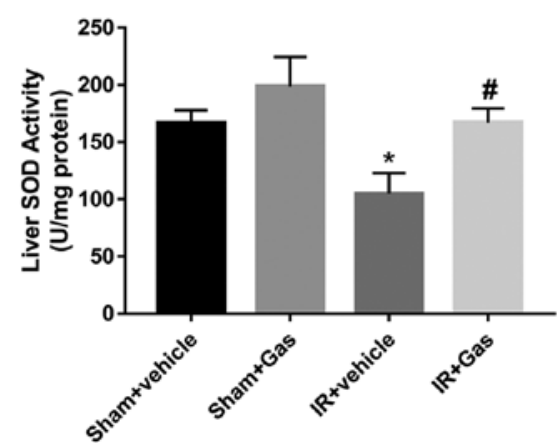

Figure 2. Gas pretreatment enhances the ability of mice to resist oxidative stress. (A) Liver tissue biochemical indexes of SOD, GSH and MDA at $2 \mathrm{~h}$ following hepatic ischemia-reperfusion modeling. (B) Liver tissue biochemical indexes of SOD, GSH and MDA at $24 \mathrm{~h}$ following hepatic IR modeling ( $\mathrm{n}=6$ mice). ${ }^{*} \mathrm{P}<0.05,{ }^{* *} \mathrm{P}<0.01,{ }^{* * * *} \mathrm{P}<0.001$ vs. the Sham + vehicle group; ${ }^{*} \mathrm{P}<0.05,{ }^{\# \#} \mathrm{P}<0.01$ vs. the IR + vehicle group. Gas, gastrodin; GSH, glutathione; IR, ischemia-reperfusion; MDA, malondialdehyde; SOD, superoxide dismutase.

during HIRI. In addition, Gas increased the content of GSH in the liver of normal mice, suggesting an increased resistance to attacks from oxygen free radicals and reactive oxygen species. This effect was consistent with previously published studies $(21,22)$.

A previous study has reported that Nrf2 regulates HO-1 expression levels by binding to antioxidative stress elements on the HO-1 promoter (23). Nrf2 protected organisms from oxidative stress by regulating the expression levels of antioxidant enzymes (24). In addition, ischemic hypoxia stimulation has been shown to induce the production of HO-1, which exerts protective antioxidant functions $(6,25,26)$. Consistent with these findings, the present experimental results revealed that Gas pretreatment upregulated the mRNA and protein expression levels of Nrf2 and HO-1 at $24 \mathrm{~h}$ post hepatic IR modeling, with the two demonstrating a similar trend. However, compared with the Sham + vehicle group at 2 and $24 \mathrm{~h}$ post IR modeling, Nrf2 mRNA and protein expression levels in the IR + vehicle group were upregulated at first, but then decreased, whereas HO-1 mRNA and protein expression levels demonstrated an upward trend. Therefore, there did not appear to be a similar trend between liver Nrf2 and HO-1 expression levels in the IR + vehicle group at the $24 \mathrm{~h}$ time-point after establishment of the model. However, pretreatment with Gas produced a similar change between Nrf2 and HO-1 expression levels at $24 \mathrm{~h}$ post modeling, rather than at $2 \mathrm{~h}$. This finding suggested that there may be another regulatory mechanism acting between Nrf2 and HO-1 under gastrodin action in the subacute time window (2-24 h) following HIRI. According to a previous study, Gas promotes the nuclear transfer of Nrf2 (20). This phenomenon may explain the experimental results; however, the associated mechanism of action requires further research. 
A

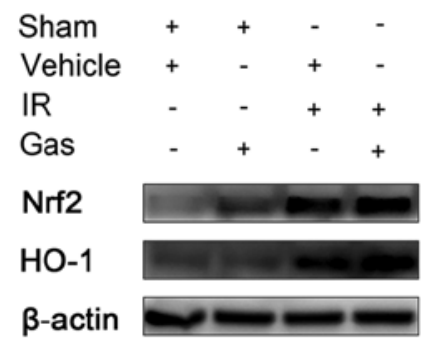

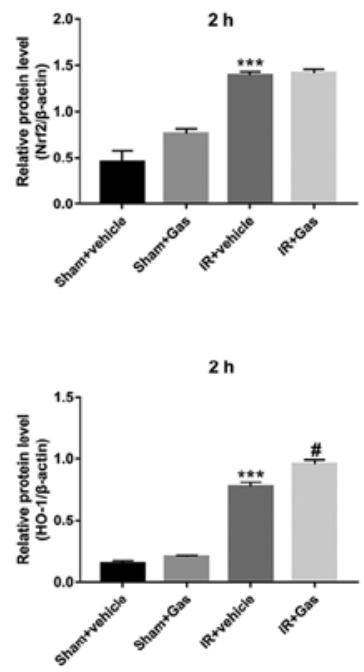

B
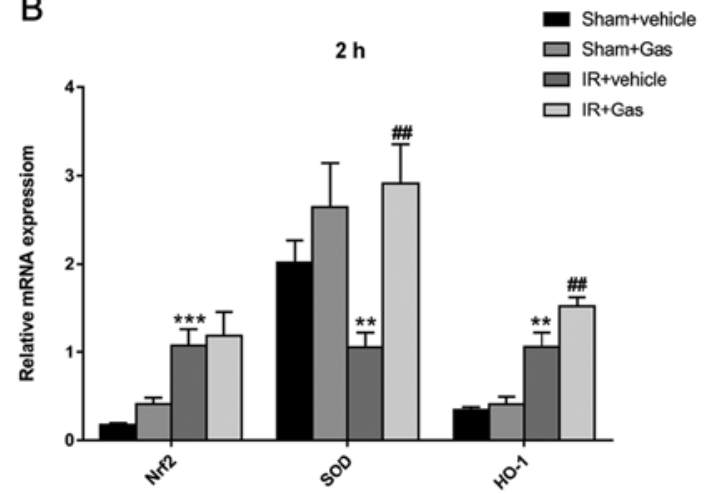

Figure 3. Gas pretreatment upregulates Nrf2 and HO-1 expression levels in the mouse liver. (A) Western blotting was used to determine Nrf2 and HO-1 protein expression levels at $2 \mathrm{~h}$ following hepatic IR modeling ( $\mathrm{n}=3$ mice). (B) Reverse transcription-quantitative PCR was used to analyze the expression levels of $\mathrm{Nrf} 2, \mathrm{HO}-1$ and SOD at $2 \mathrm{~h}$ post hepatic IR modeling $(\mathrm{n}=6) .{ }^{* * *} \mathrm{P}<0.01,{ }^{* * *} \mathrm{P}<0.001$ vs. the Sham + vehicle group; ${ }^{*} \mathrm{P}<0.05$, ${ }^{\# *} \mathrm{P}<0.01$ vs. the IR + vehicle group. Gas, gastrodin; IR, ischemia-reperfusion; Nrf2, nuclear erythroid 2-related factor 2; HO-1, heme oxygenase-1; SOD, superoxide dismutase.
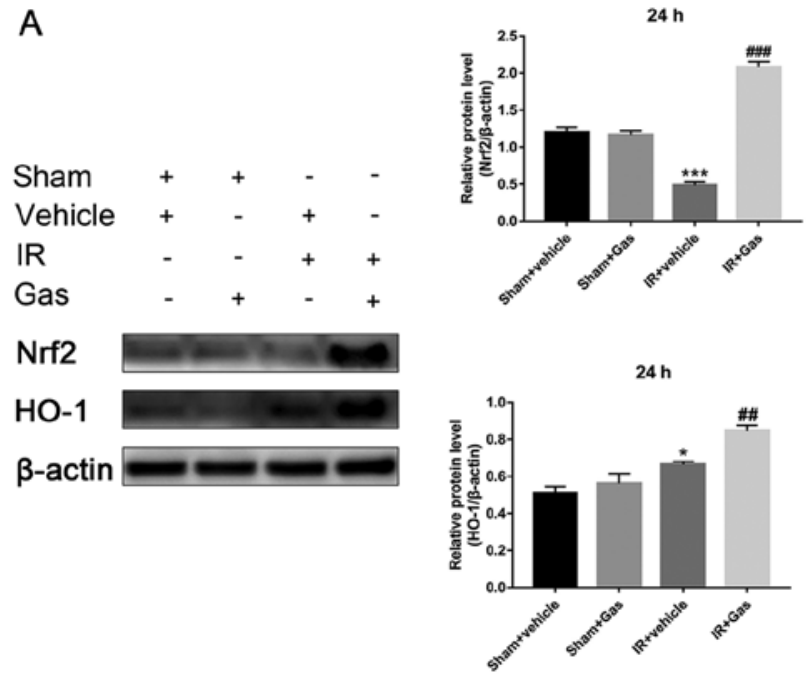

B

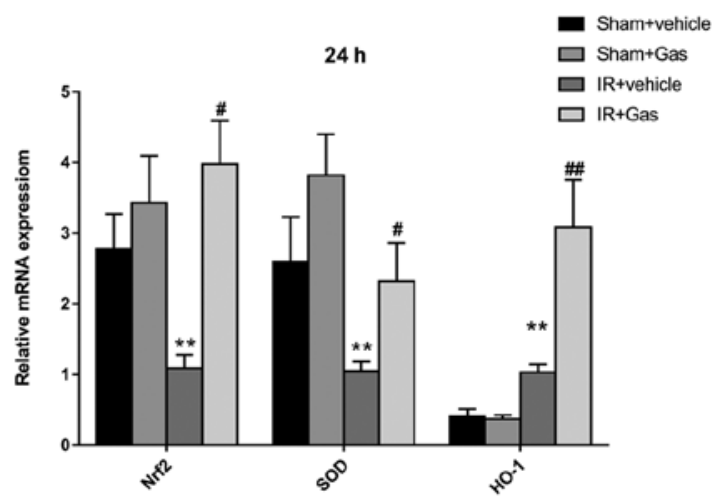

Figure 4. Gas pretreatment upregulates Nrf2 and HO-1 expression levels in the mice liver. (A) Western blotting was used to determine Nrf2 and HO-1 protein expression levels at $24 \mathrm{~h}$ post hepatic IR modeling $(\mathrm{n}=3$ mice). (B) Reverse transcription-quantitative PCR was used to analyze the expression levels of Nrf 2 and HO-1 at $24 \mathrm{~h}$ post hepatic IR modeling $\left(\mathrm{n}=6\right.$ mice). ${ }^{*} \mathrm{P}<0.05,{ }^{* * *} \mathrm{P}<0.01,{ }^{* * *} \mathrm{P}<0.001$ vs. the Sham + vehicle group; ${ }^{\prime \prime} \mathrm{P}<0.05,{ }^{\# \#} \mathrm{P}<0.01,{ }^{\# \# "} \mathrm{P}<0.001$ vs. the IR + vehicle group. Gas, gastrodin; HO-1, heme oxygenase-1; IR, ischemia-reperfusion; Nrf2, nuclear erythroid 2-related factor 2; SOD, superoxide dismutase.

Necrosis has been considered as the main outcome of dying tissue cells from acute injury. However, this conclusion has not been confirmed after inhibition at key regulatory sites during necrosis in acute liver injury models (27). Concurrently, the process of apoptosis, another form of cell death, has attracted increasing attention. HIRI was significantly improved by inhibiting proapoptotic molecules, since apoptosis is one of the predominant forms of liver cell death (28). Previous studies have revealed that by inhibiting c-Jun NH2 terminal kinase 2, the release of mitochondrial proapoptotic molecules was decreased $(29,30)$, thereby reducing the apoptosis of hepatocytes and markedly improving adverse IR events (3). In addition, Bax and Bcl-2 regulate cell survival or resistance by promoting signal transduction (4). CASP-3 exists as a precursor and functions following its cleavage to cleaved CASP-3 (31). Furthermore, the expression levels of CASP-3 mRNA were identified to be upregulated in association with the occurrence of apoptosis (32). Therefore, the present study investigated the relationship between Gas and the expression levels of the apoptosis-related proteins, Bax, Bcl-2 and CASP-3. The experimental results revealed that Gas pretreatment may reduce the incidence of hepatocyte apoptosis.

In conclusion, the findings of the present study revealed that Gas pretreatment increased the content of GSH in the liver, enhanced the activity of SOD, upregulated the expression levels of antioxidant-related genes Nrf2 and HO-1, and regulated the expression levels of apoptosis-related proteins, $\mathrm{Bax}, \mathrm{Bcl}-2$ and CASP-3, thereby significantly alleviating HIRI. These results may provide insights into a novel therapeutic strategy for the 


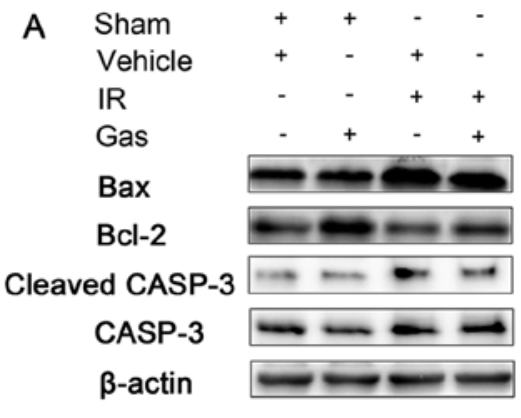

2h

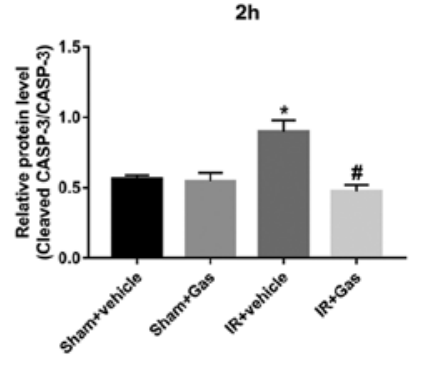

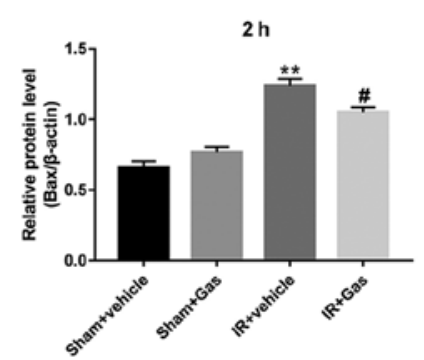

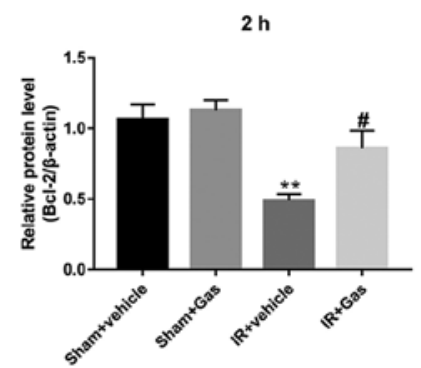

B
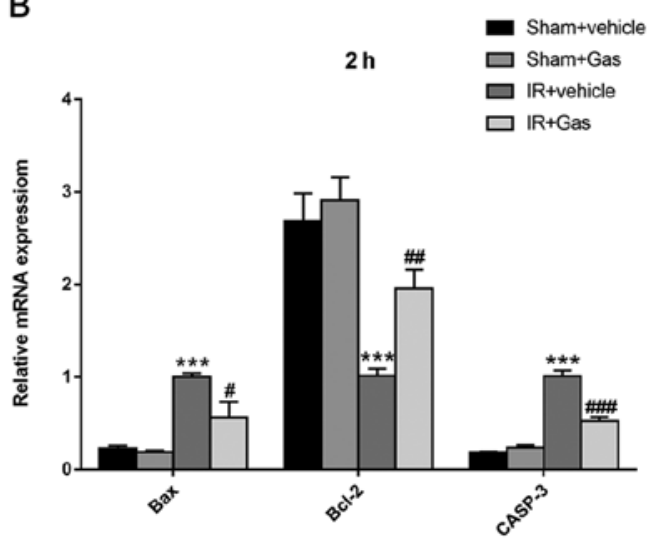

Figure 5. Gas pretreatment improves the antiapoptotic ability of the liver through regulating the expression levels of Bax, Bcl-2 and CASP-3. (A) Protein expression levels of Bax, Bcl-2 and CASP-3 at $2 \mathrm{~h}$ following hepatic IR modeling were analyzed using western blotting ( $\mathrm{n}=3$ ). (B) RT-qPCR was used to analyze the expression levels of Bax, Bcl-2 and CASP-3 at $2 \mathrm{~h}$ post hepatic ischemic-reperfusion modeling ( $\mathrm{n}=6 \mathrm{mice}){ }^{*} \mathrm{P}<0.05,{ }^{* *} \mathrm{P}<0.01,{ }^{* * *} \mathrm{P}<0.001 \mathrm{vs}$. the Sham + vehicle group; ${ }^{\#} \mathrm{P}<0.05,{ }^{\#} \mathrm{P}<0.01,{ }^{\# \#} \mathrm{P}<0.001$ vs. the IR + vehicle group. CASP-3, caspase 3; Gas, gastrodin; IR, ischemia-reperfusion; RT-qPCR, reverse transcription-quantitative $\mathrm{PCR}$.
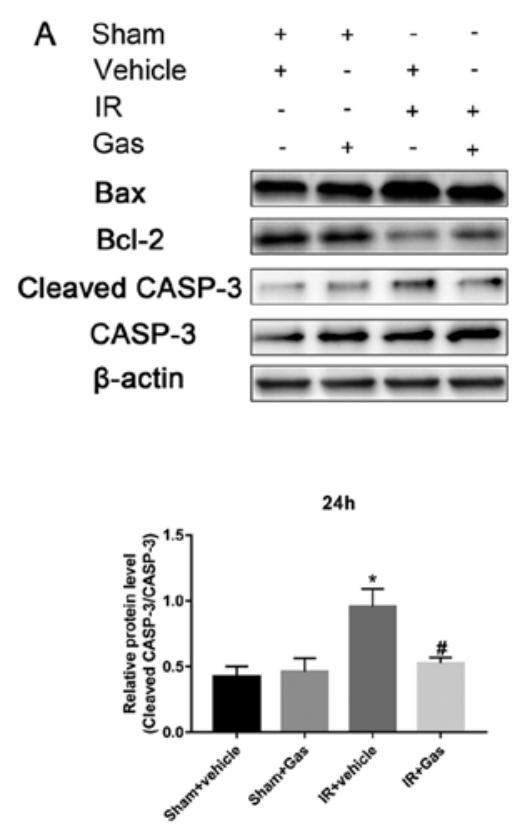
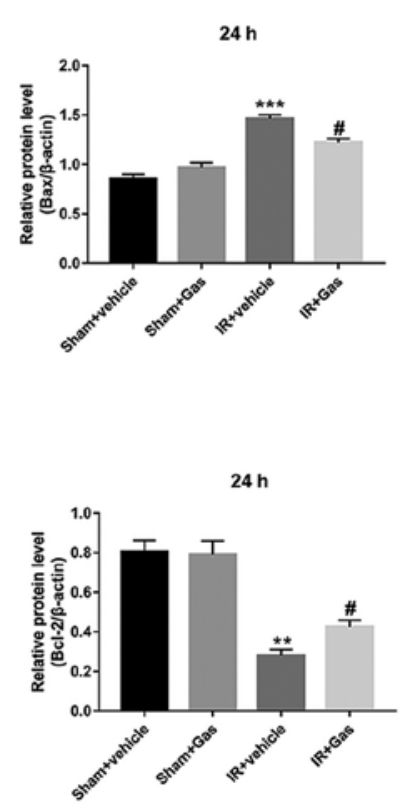

B

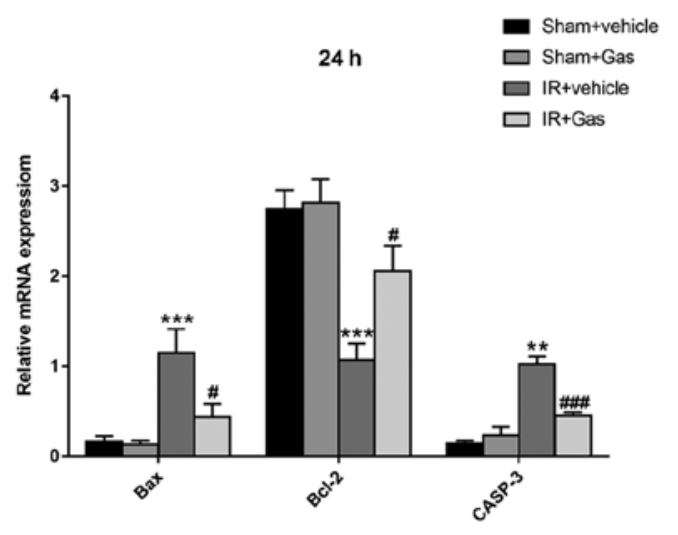

Figure 6. Gas pretreatment improves the antiapoptotic ability of the liver through regulating the expression levels of Bax, Bcl-2 and CASP-3. (A) Western blotting analysis of Bax, Bcl-2 and CASP-3 protein expression levels at $24 \mathrm{~h}$ post hepatic IR modeling (n=3). (B) Reverse transcription-quantitative PCR analysis of Bax, Bcl-2 and CASP-3 mRNA expression levels at $24 \mathrm{~h}$ post hepatic IR modeling $(\mathrm{n}=6 \mathrm{mice}){ }^{*} \mathrm{P}<0.05,{ }^{* *} \mathrm{P}<0.01,{ }^{* * *} \mathrm{P}<0.001 \mathrm{vs}$. the Sham + vehicle group; ${ }^{\#} \mathrm{P}<0.05,{ }^{\# \#} \mathrm{P}<0.001$ vs. the IR + vehicle group. CASP-3, caspase 3; Gas, gastrodin; IR, ischemia-reperfusion; RT-qPCR, reverse transcription-quantitative PCR.

clinical treatment and prevention of liver failure following liver surgery.

\section{Acknowledgements}

The authors would like to thank pathologists (Dr Xuyou Zhu, Dr Tingting Zhang and Dr Yu Zeng) of the Pathology
Department of Shanghai Tongji Hospital, Shanghai, China, for scoring the experimental samples.

\section{Funding}

The present study was supported by grants from the National Natural Science Foundation of China (grant no. 81873567) and 
the Shanghai Municipal Commission of Health and Family Planning (grant no. WSJ1607).

\section{Availability of data and materials}

The data used and/or analyzed during the current study are available from the corresponding author on reasonable request.

\section{Authors' contributions}

WY and RL conceived and designed the study. BS, JJ and $\mathrm{XZ}$ collected the tissue samples and established the mouse model. DY, ZC, YZ, MZ and YQ performed the experiments and acquired the data. BS and WY confirm the authenticity of all the raw data. WY, RL, XZ, BS and JJ interpreted the data and prepared and revised the manuscript. All authors read and approved the final manuscript.

\section{Ethics approval and consent to participate}

The present studywas approved by the AnimalEthics Committee of Tongji University (approval no. TJHBLAC-2019-116; Shanghai, China).

\section{Patient consent for publication}

Not applicable.

\section{Competing interests}

The authors declare that they have no competing interests.

\section{References}

1. Dery KJ, Nakamura K, Kadono K, Hirao H, Kageyama S, Ito T, Kojima H, Kaldas FM, Busuttil RW and Kupiec-Weglinski JW: Human Antigen R (HuR): A new regulator of heme oxygenase-1 cytoprotection in mouse and human liver transplant injury. Hepatology 72: 1056-1072, 2019.

2. Covington SM, Bauler LD and Toledo-Pereyra LH: Akt: A therapeutic target in hepatic ischemia-reperfusion injury. J Invest Surg 30: 47-55, 2017.

3. Peralta C, Jimenez-Castro MB and Gracia-Sancho J: Hepatic ischemia and reperfusion injury: Effects on the liver sinusoidal milieu. J Hepatol 59: 1094-1106, 2013.

4. Han X, Shi H, Liu K, Zhong L, Wang F and You Q: Protective effect of gastrodin on myocardial ischemia-reperfusion injury and the expression of Bax and Bcl-2. Exp Ther Med 17: 4389-4394, 2019.

5. Ju C, Colgan SP and Eltzschig HK: Hypoxia-inducible factors as molecular targets for liver diseases. J Mol Med (Berl) 94: 613-627, 2016.

6. Qiu M, Xiao F, Wang T, Piao S, Zhao W, Shao S, Yan M and Zhao D: Protective effect of Hedansanqi Tiaozhi Tang against non-alcoholic fatty liver disease in vitro and in vivo through activating $\mathrm{Nrf} 2 / \mathrm{HO}-1$ antioxidant signaling pathway. Phytomedicine 67: 153140, 2019.

7. Theruvath TP, Czerny C, Ramshesh VK, Zhong Z, Chavin KD and Lemasters JJ: C-Jun N-terminal kinase 2 promotes graft injury via the mitochondrial permeability transition after mouse liver transplantation. Am J Transplant 8: 1819-1828, 2008.

8. Matias M, Silvestre S, Falcao A and Alves G: Gastrodia elata and epilepsy: Rationale and therapeutic potential. Phytomedicine 23: 1511-1526, 2016

9. Li JJ, Liu SJ, Liu XY and Ling EA: Herbal compounds with special reference to gastrodin as potential therapeutic agents for microglia mediated neuroinflammation. Curr Med Chem 25: 5958-5974, 2018.

10. $\mathrm{Hu}$ Y, Li C and Shen W: Gastrodin alleviates memory deficits and reduces neuropathology in a mouse model of Alzheimer's disease. Neuropathology 34: 370-377, 2014.
11. Matsumata T, Kanematsu T, Shirabe K, Yamagata M, Utsunomiya T, Furuta T and Sugimachi K: Modified technique of pringle's maneuver in resection of the liver. Surg Gynecol Obstet 172: 245-246, 1991.

12. Li Y, Li T and Qi H: Protective effect of minocycline on hepatic ischemia-reperfusion injury in rats. Zhong Nan Da Xue Xue Bao Yi Xue Ban 39: 1137-1144, 2014 (In Chinese).

13. Livak KJ and Schmittgen TD: Analysis of relative gene expression data using real-time quantitative PCR and the 2(-Delta Delta C(T)) method. Methods 25: 402-408, 2001.

14. Smedsrød B, De Bleser PJ, Braet F, Lovisetti P, Vanderkerken K, Wisse E and Geerts A: Cell biology of liver endothelial and Kupffer cells. Gut 35: 1509-1516, 1994.

15. Kaplowitz N: Drug-induced liver injury. Clin Infect Dis 38 (Suppl 2): S44-S48, 2004.

16. Senoner T, Schindler S, Stattner S, Ofner D, Troppmair J and Primavesi F: Associations of oxidative stress and postoperative outcome in liver surgery with an outlook to future potential therapeutic options. Oxid Med Cell Longev 2019: 3950818, 2019.

17. Yang P, Han Y, Gui L, Sun J, Chen YL, Song R, Guo JZ, Xie YN, $\mathrm{Lu} \mathrm{D}$ and Sun L: Gastrodin attenuation of the inflammatory response in $\mathrm{H} 9 \mathrm{c} 2$ cardiomyocytes involves inhibition of $\mathrm{NF}-\mathrm{kB}$ and MAPKs activation via the phosphatidylinositol 3-kinase signaling. Biochem Pharmacol 85: 1124-1133, 2013.

18. Chen Pz, Jiang Hh, Wen B, Ren SC, Chen Y, Ji WG, Hu B, Zhang J, Xu F and Zhu ZR: Gastrodin suppresses the amyloid $\beta$-induced increase of spontaneous discharge in the entorhinal cortex of rats. Neural Plast 2014: 320937, 2014.

19. Zhan HD, Zhou HY, Sui YP, Du XL, Wang WH, Dai L, Sui F, Huo HR and Jiang TL: The rhizome of Gastrodia elata Blume-An ethnopharmacological review. J Ethnopharmacol 189: 361-385, 2016.

20. Qu LL, Yu B, Li Z, Jiang WX, Jiang JD and Kong WJ: Gastrodin ameliorates oxidative stress and proinflammatory response in nonalcoholic fatty liver disease through the AMPK/Nrf2 pathway. Phytother Res 30: 402-411, 2016.

21. Dai JN, Zong Y, Zhong LM, Li YM, Zhang W, Bian LG, Ai QL, Liu YD, Sun J and Lu D: Gastrodin inhibits expression of inducible NO synthase, cyclooxygenase-2 and proinflammatory cytokines in cultured LPS-stimulated microglia via MAPK pathways. PLoS One 6: e21891, 2011.

22. Peng Z, Wang S, Chen G, Cai M, Liu R, Deng J, Liu J, Zhang T, Tan Q and Hai C: Gastrodin alleviates cerebral ischemic damage in mice by improving anti-oxidant and anti-inflammation activities and inhibiting apoptosis pathway. Neurochem Res 40: 661-673, 2015.

23. Masuda Y, Vaziri ND, Takasu C, Li S, Robles L, Pham C, Le A, Vo K, Farzaneh SH, Stamos MJ and Ichii H: Salutary effect of pre-treatment with an Nrf2 inducer on ischemia reperfusion injury in the rat liver. Gastroenterol Hepatol (Que) 1: 1-7, 2014.

24. Chen Z, Zhong H, Wei J, Lin S, Zong Z, Gong F, Huang X, Sun J, $\mathrm{Li}$, Lin $\mathrm{H}$, et al: Inhibition of Nrf2/HO-1 signaling leads to increased activation of the NLRP3 inflammasome in osteoarthritis. Arthritis Res Ther 21: 300, 2019.

25. Tanaka Y, Maher JM, Chen C and Klaassen CD: Hepatic ischemia-reperfusion induces renal heme oxygenase-1 via NF-E2-related factor 2 in rats and mice. Mol Pharmacol 71: 817-825, 2007.

26. Bauer M and Bauer I: Heme oxygenase-1: Redox regulation and role in the hepatic response to oxidative stress. Antioxid Redox Signal 4: 749-758, 2002.

27. Schwabe RF and Luedde T: Apoptosis and necroptosis in the liver: A matter of life and death. Nat Rev Gastroenterol Hepatol 15: 738-752, 2018.

28. Lei K and Davis RJ: JNK phosphorylation of Bim-related members of the Bcl2 family induces Bax-dependent apoptosis. Proc Natl Acad Sci USA 100: 2432-2437, 2003.

29. Tournier C, Hess P, Yang DD, Xu J, Turner TK, Nimnual A, Bar-Sagi D, Jones SN, Flavell RA and Davis RJ: Requirement of JNK for stress-induced activation of the cytochrome c-mediated death pathway. Science 288: 870-874, 2000.

30. Liu Q, Rehman H, Krishnasamy Y, Schnellmann RG, Lemasters JJ and Zhong Z: Improvement of liver injury and survival by JNK2 and iNOS deficiency in liver transplants from cardiac death mice. J Hepatol 63: 68-74, 2015.

31. Shalini S, Dorstyn L, Dawar S and Kumar S: Old, new and emerging functions of caspases. Cell Death Differ 22: 526-539, 2015.

32. Lossi L, Castagna C and Merighi A: Caspase-3 mediated cell death in the normal development of the mammalian cerebellum. Int J Mol Sci 19: 3999, 2018. 